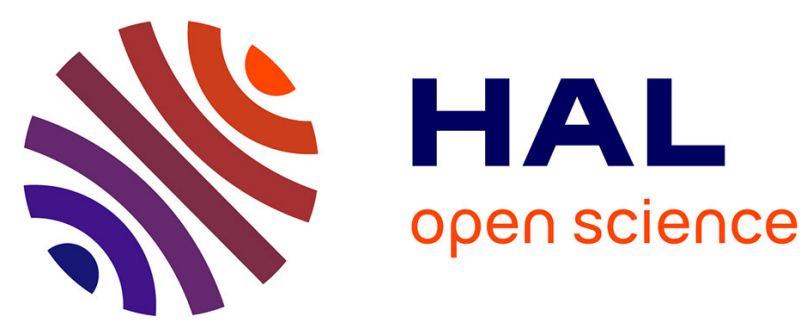

\title{
Une sépulture à incinération sur l'oppidum de Mourrel-Ferrat à Olonzac (Hérault). Fin premier âge du Fer - début second âge du Fer.
}

Pierre Séjalon, Laurent Bouby, Nathalie Chardenon, Armelle Gardeisen, Thierry Janin

\section{To cite this version:}

Pierre Séjalon, Laurent Bouby, Nathalie Chardenon, Armelle Gardeisen, Thierry Janin. Une sépulture à incinération sur l'oppidum de Mourrel-Ferrat à Olonzac (Hérault). Fin premier âge du Fer - début second âge du Fer.. Documents d'archéologie méridionale, 2000, 23, pp.209-217. halshs-01421188

\author{
HAL Id: halshs-01421188 \\ https://shs.hal.science/halshs-01421188
}

Submitted on 29 Dec 2016

HAL is a multi-disciplinary open access archive for the deposit and dissemination of scientific research documents, whether they are published or not. The documents may come from teaching and research institutions in France or abroad, or from public or private research centers.
L'archive ouverte pluridisciplinaire HAL, est destinée au dépôt et à la diffusion de documents scientifiques de niveau recherche, publiés ou non, émanant des établissements d'enseignement et de recherche français ou étrangers, des laboratoires publics ou privés. 


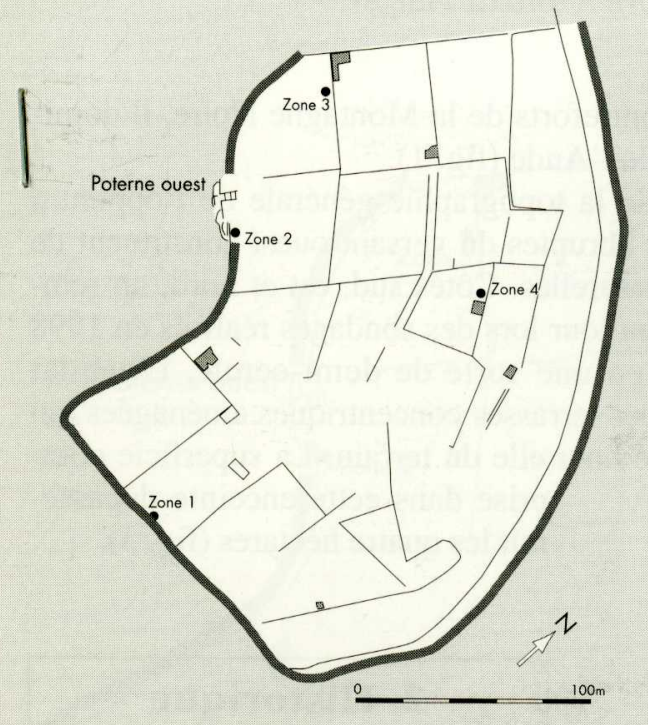

\title{
Une sépulture à incinération sur l'oppidum de Mourrel-Ferrat à Olonzac (Hérault)
}

\section{(fin premier âge du Fer - début second âge du Fer)}

\author{
Pierre SÉJALON * \\ en collaboration avec Laurent BOUBY **, \\ Nathalie CHARDENON *, Armelle GARDEISEN *, Thierry JANIN *
}

\begin{abstract}
C'est à l'occasion d'une campagne de sondages effectuée afin de préciser la chronologie de l'oppidum de Mourrel-Ferrat (Hérault) et de dater l'aménagement de murs et de talus imposants, qu'une sépulture à incinération a été mise au jour. Elle se présente sous la forme d'un loculus creusé dans le calcaire gélifracté. Le dépôt caractérisé par une poignée d'os humains brûlés, des esquilles fauniques et un os de cerf, des graines brûlées, un couteau en fer, un fragment de panse d'amphore massaliète et quelques tessons informes de céramique non tournée, était localisé dans le tiers supérieur du remplissage avec une superposition de certains éléments. Cette découverte qui pourrait signaler la présence d'une nécropole au sein de la fortification ouvre de séduisantes perspectives quant à l'approche des pratiques funéraires de la fin du premier âge du Fer et du début du second qui sont encore largement méconnues.
\end{abstract}

Mots-clés : sépulture, incinération, âge du fer, Protohistoire, oppidum.
A cremation was discovered during a programme of trial trenching that aimed to ascertain the chronology of the Mourrel-Ferrat oppidum (Herault) and to date the walls and embankments. The cremation was found in a pit dug into the gelifracted limestone. In the upper layers of the deposit the following elements were discovered : a handful of burnt human bones, plus splinters of animal bones, a deer bone, burnt grain, an iron knife, a fragment of an massaliete amphorae handle plus some shards of hand-thrown pottery. This discovery could indicate that there was cemetery within the fortifications, thus supplying us with some interesting perspectives vis a vis funerary practices at the end of the early and the beginning of the late Iron Ages.

Key-words : Burial, cremation, Iron Age, Protohistory, oppidum. 


\section{Situation géographique}

9ppidum de Mourrel-Ferrat se trouve à une dizaine de kilomètres au sud-est de la commune d'Olonzac (Hérault). Son développement le plus étendu chevauche les départements de l'Aude et de l'Hérault. Implanté à l'extrémité d'une ligne de collines qui sépare la plaine narbonnai-

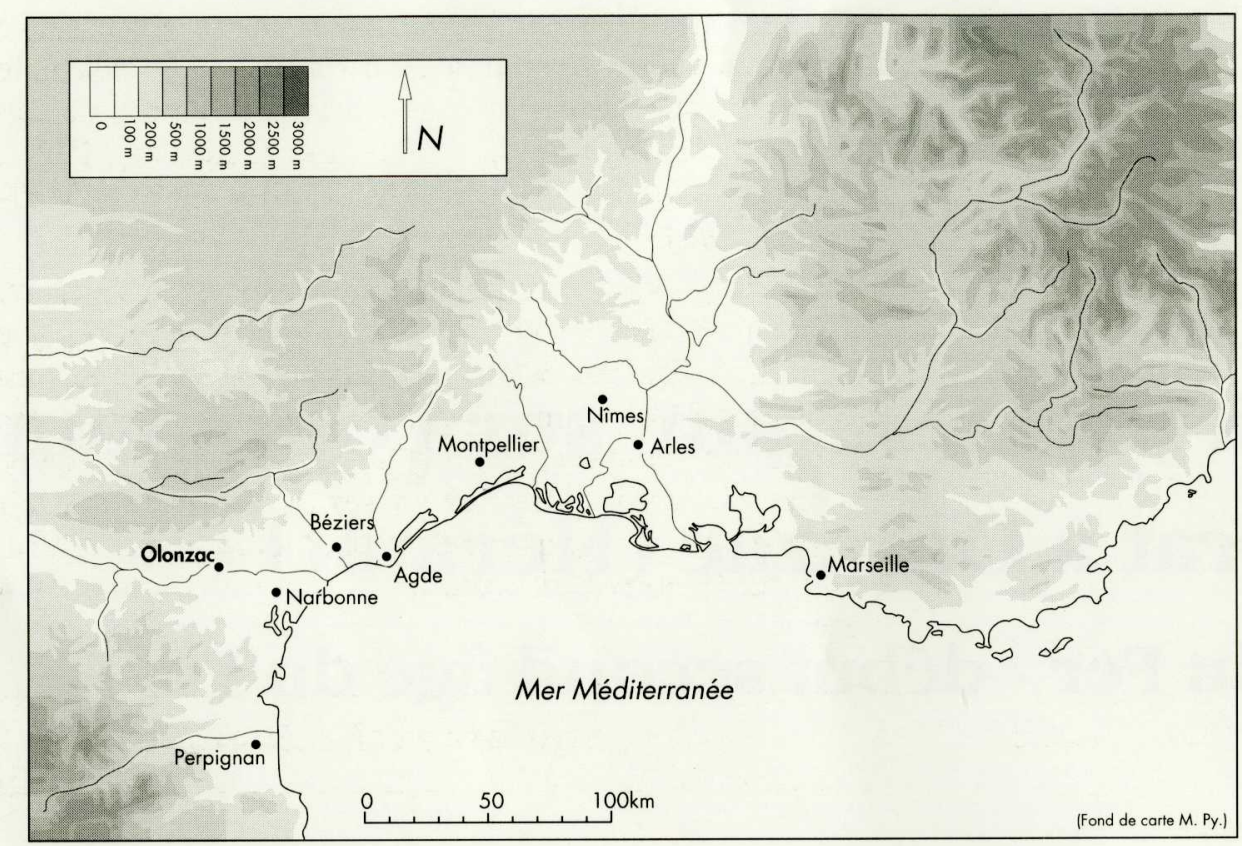

1 Localisation de la commune d'Olonzac (Hérault).

12 Situation topographique de l'oppidum de Mourrel-Ferrat d'après la carte IGN 2545 E (25000, 1982).

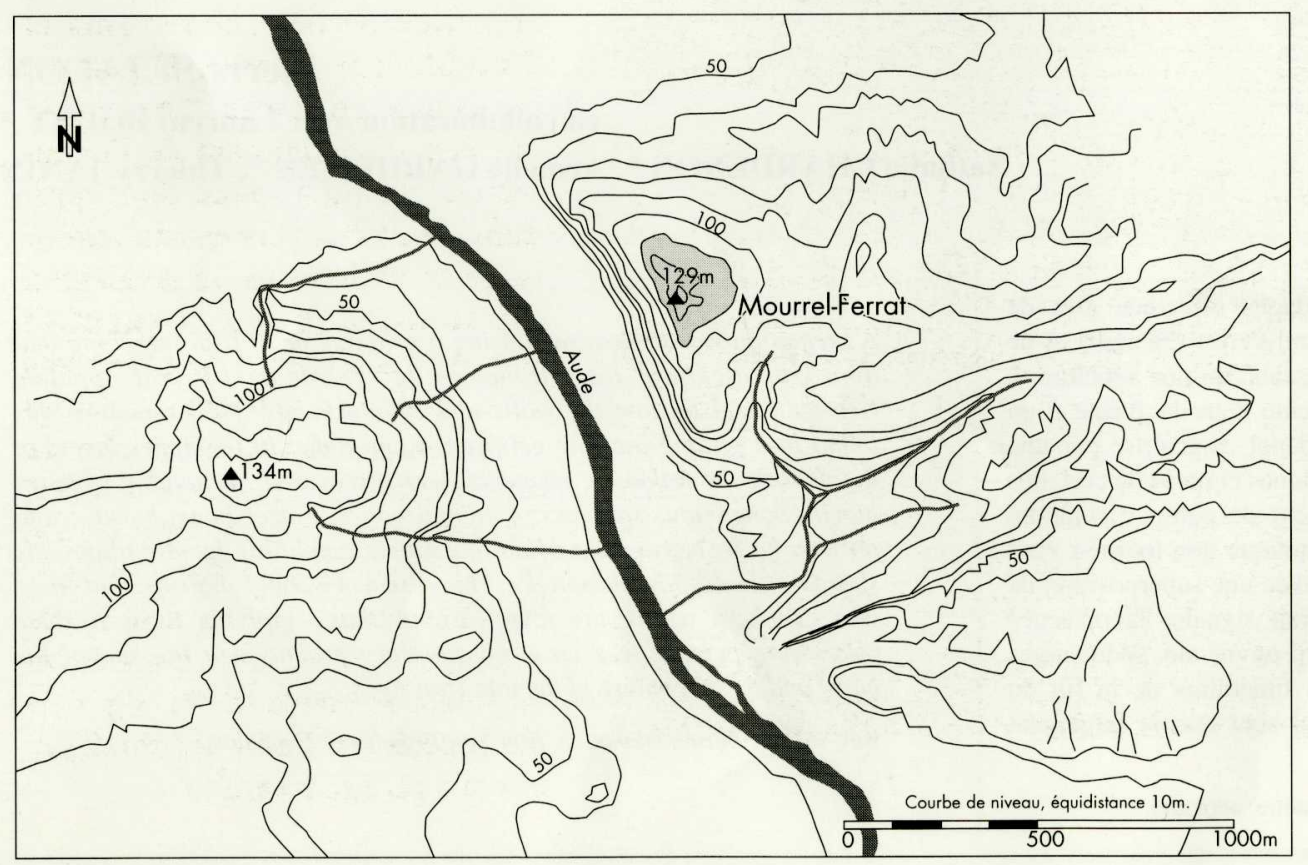

se des premiers contreforts de la Montagne Noire, il domine la rive gauche de l'Aude (fig. 1).

Du point de vue de la topographie générale de l'oppidum (fig. 2), les pentes abruptes du versant ouest constituent de bonnes défenses naturelles. Côtés sud, est et nord, un rempart (zone 1) mis au jour lors des sondages réalisés en 1998 ferme le plateau en une sorte de demi-cercle. L'habitat semble établi sur des terrasses concentriques aménagées qui suivent la déclivité naturelle du terrain. La superficie comprise dans cette enceinte dépasserait les quatre hectares (fig. 3).

\section{Historique des recherches}

D après les publications relatives au site, c'est L. Mathieu qui a mené les premières explorations archéologiques sur l'oppidum (Mathieu 1931).

Par la suite, Y. Solier a pratiqué un sondage le long du rempart ouest à proximité de la poterne dégagée par le CRDM (Centre de Documentation et de Recherche du Minervois) (CRDM 1980) dont les résultats sont inédits. Les derniers travaux sont ceux effectués par les membres du CRDM sous la direction de Maurice Rigal en 1987. Ils ont permis la fouille d'une habitation complète sans doute détruite par un incendie et datée de la fin du $\mathrm{IV}^{\mathrm{e}}$ - début du $\mathrm{III}^{\mathrm{e}}$ s. av. n. è. (CRDM 1987).

Ainsi, à partir des données publiées et de celles enregistrées lors des derniers sondages, on peut cerner chronologiquement l'occupation du site entre la fin du $\mathrm{VI}^{\mathrm{e}}$ et le tout début du III $^{\mathrm{e}} \mathrm{s}$. av. n. è. Dans l'état actuel des recherches, l'oppidum serait détruit à la fin du $\mathrm{III}^{\mathrm{e}}$ s. av. n. è.; aucune installation postérieure n'est attestée exceptés des aménagements modernes, garennes et murs de mises en terrasses sans que l'on puisse préciser leur fonction (culture ou parcage de bétail). 


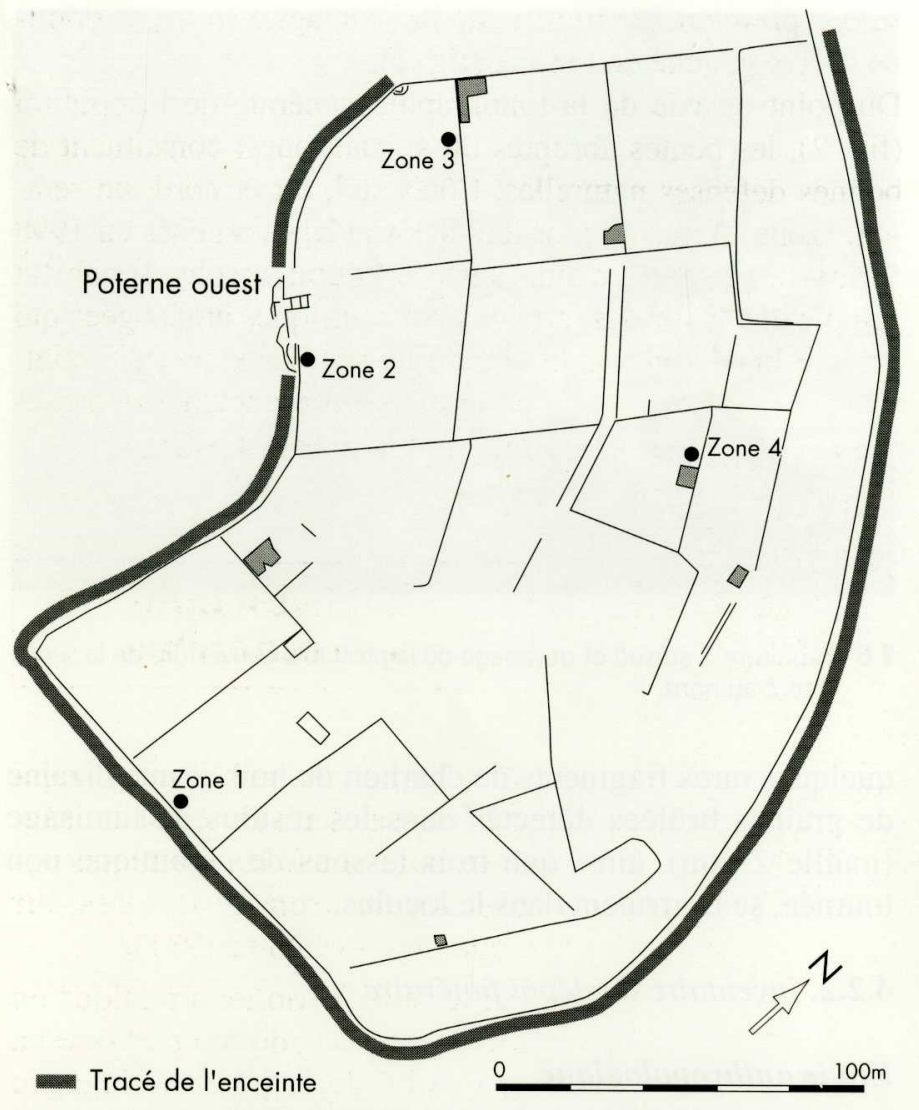

13 Plan de l'oppidum (d'après M. Rigal, 1977, CRDM) avec la localisation des sondages.

\section{Conditions de la découverte et protocole d'intervention}

E n 1997, une demande de sondages a été faite au Service Régional de l'Archéologie afin de préciser la durée d'occupation de l'oppidum de Mourrel-Ferrat ${ }^{1}$ et de dater l'aménagement de murs et de talus d'importance variable. Pour se faire, quatre sondages réduits $(2 \times 2 \mathrm{~m})$ ont été effectués (fig. 3) ${ }^{2}$. La mise au jour d'une sépulture et la probable présence d'une seconde dans un des sondages (zone 4) ont motivé la publication de cet article. Cet ensemble complète modestement la courte liste des tombes découvertes sur les oppidums : Ensérune (Jannoray 1955; Schwaller et al. 1995), Pech Maho (Solier 1968) et Mailhac (Taffanel 1960).

Afin d'enregistrer le maximum d'informations, la sépulture a été fouillée selon les protocoles de règle en anthropologie de terrain ${ }^{3}$. Tous les éléments présents dans le loculus ont été cotés en trois dimensions. La sépulture n'ayant pas livré de vase ossuaire, la fosse a d'emblée été considérée comme tel. Ainsi, toutes les phases de décapage ont fait l'objet de relevés graphiques et photographiques. Enfin, l'intégralité du remplissage de la fosse a été tamisée à l'eau.

\section{Les résultats archéologiques}

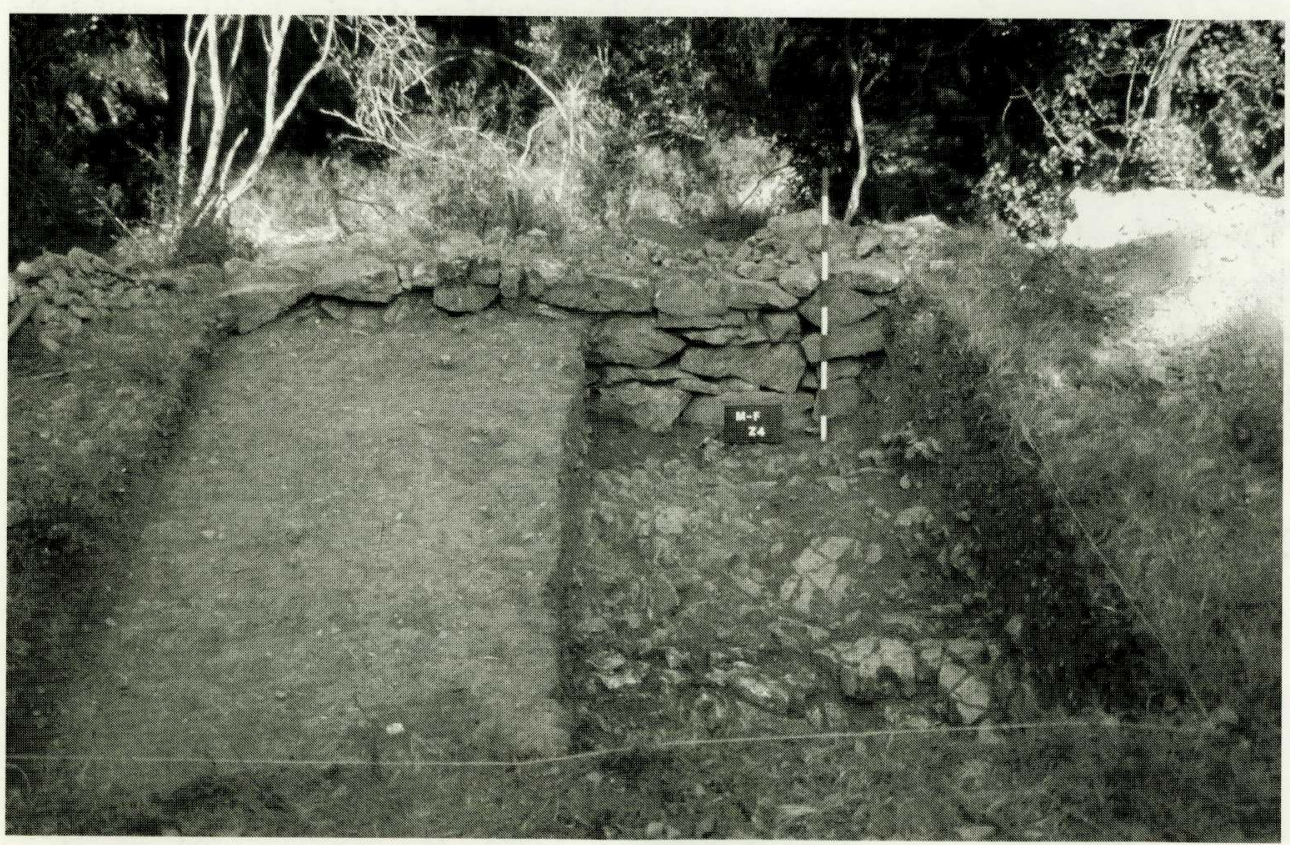

14 Vue générale de la zone 4.

\subsection{LA ZONE 4}

Ce sondage a été implanté contre un large mur ( $1 \mathrm{~m}$ d'épaisseur) constitué de deux parements et d'un blocage interne. Il marque une courbe de niveau et ne semble pas être contemporain de l'occupation protohistorique de l'oppidum. Pour des raisons de temps, seule la moitié sud du sondage a été fouillée jusqu'au substrat (fig. 4). La présence de fragments de céramique non tournée calcinés emprisonnés dans une poche de terre a attiré notre attention. C'est en décapant cette anfractuosité que les premiers os humains brûlés et le couteau en fer sont apparus (fig. 5). A proximité, une autre sépulture probable (SP2) (fig. 6) a été repérée en limite du sondage; elle n'a pu être fouillée. 


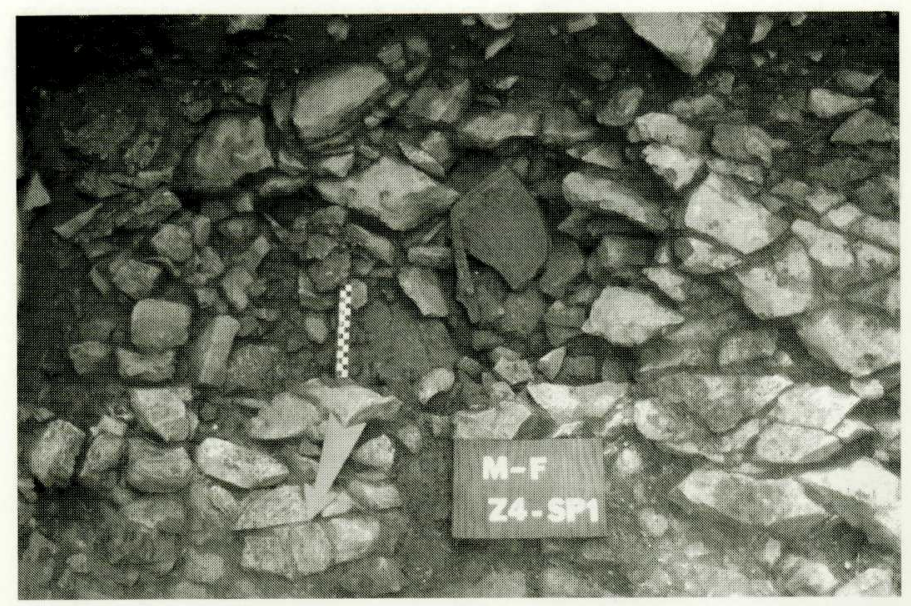

I 5 Premier décapage de la sépulture 1. Apparition du couteau en fer reposant sur le fragment de panse d'amphore massaliète.

\subsection{LA SÉPULTURE 1 (SP1)}

\subsubsection{Description générale}

Le loculus de la tombe est creusé dans le substrat calcaire (fig. 7). L'état gélifracté de ce dernier a probablement facilité l'aménagement de la fosse sans toutefois permettre un creusement régulier. La fosse mesure à l'ouverture $0,40 \times$ $0,25 \mathrm{~m}$ pour une profondeur maximale de $0,40 \mathrm{~m}$.

Le remplissage de la fosse, composé de terre et de pierres de différents modules, était vierge de cendre. Seuls

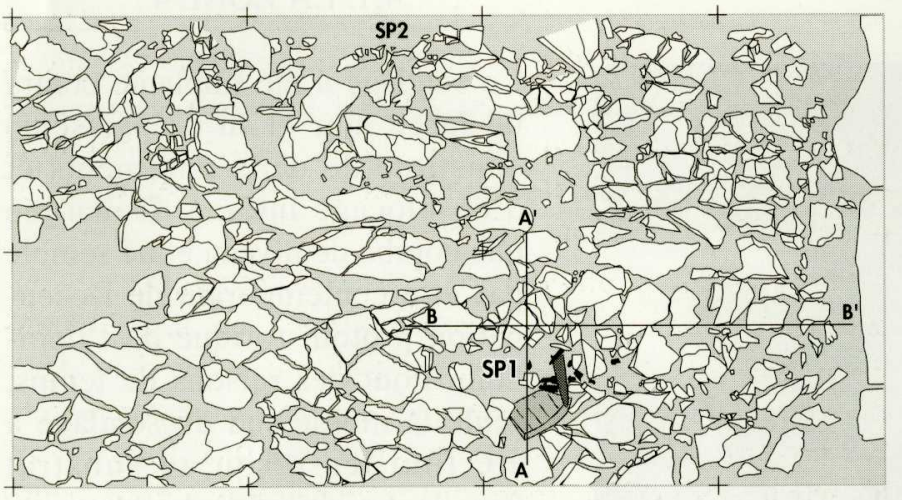

6ragment d'amphore massaliète

1 Couteau en fer

* Ossements humains brûlés

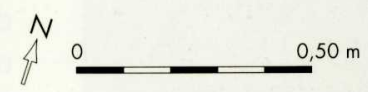

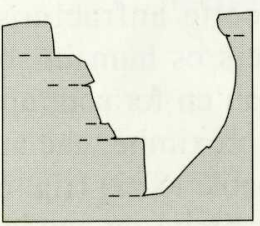

Coupe $A A^{\prime}$

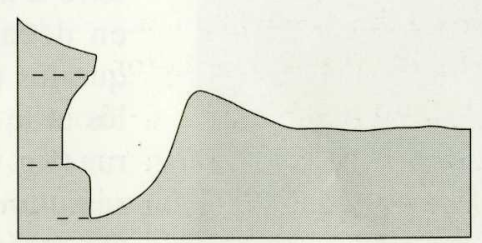

Coupe BB' $^{\prime}$ $\square 7$ Relevé de la zone 4 fouillée et coupes de la sépulture 1

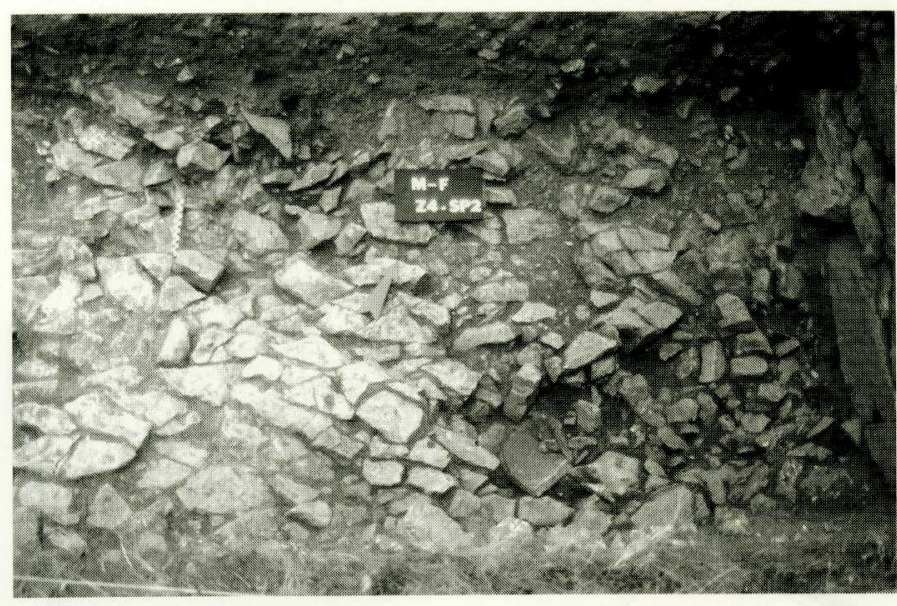

- 6 Sépulture 1 au sud et décapage de la probable couverture de la sépulture 2 au nord.

quelques rares fragments de charbon de bois et une dizaine de graines brûlées détectés dans les résidus de tamisage (maille $2 \mathrm{~mm}$ ), ainsi que trois tessons de céramique non tournée, se trouvaient dans le loculus.

\subsubsection{Inventaire du dépôt funéraire}

\section{Etude anthropologique}

La fouille du loculus et le tamisage des sédiments ont permis de recueillir au total $12,4 \mathrm{~g}$ d'ossements humains brûlés. Ils appartiennent à un sujet d'âge indéterminé, mais pas un nouveau-né. Aucune pièce n'est vraiment identifiable et on a seulement pu rapporter les fragments aux diverses régions anatomiques. La très grande majorité des fragments appartient aux os des membres; de rares os du tronc ont été recensés et deux fragments se rapportant peut-être à la tête (?) ont été identifiés. Les indices pondéraux, qui expriment en $\%$ le poids des fragments de chaque région anatomique rapporté au poids total des vestiges humains (Duday 1989 , 465), sont à ce propos éloquents (fig. 8).

La répartition des fragments dans la fosse n'apporte aucune indication particulière; on signalera cependant la distribution verticale des vestiges en deux groupes, sans que cela implique réellement un geste funéraire délibéré ou la présence possible de deux sépultures. Un fragment du lot supérieur $\left(n^{\circ} 6\right)$ colle d'ailleurs avec un élément situé dans la moitié inférieure du loculus ( $\mathrm{n}^{\circ} 37$ ) (fig. 9). On n'envisage-

\begin{tabular}{|c|c|c|}
\hline Région & Poids & Indice Pondéral \\
\hline Tête & 0,1 & 1 \\
\hline Tronc & 0,6 & 5 \\
\hline Membres & 11,1 & 89 \\
\hline Indéterminés & 0,6 & 5 \\
\hline Total & 12,4 & 100 \\
\hline
\end{tabular}

18 Indices pondéraux des différentes régions anatomiques. 


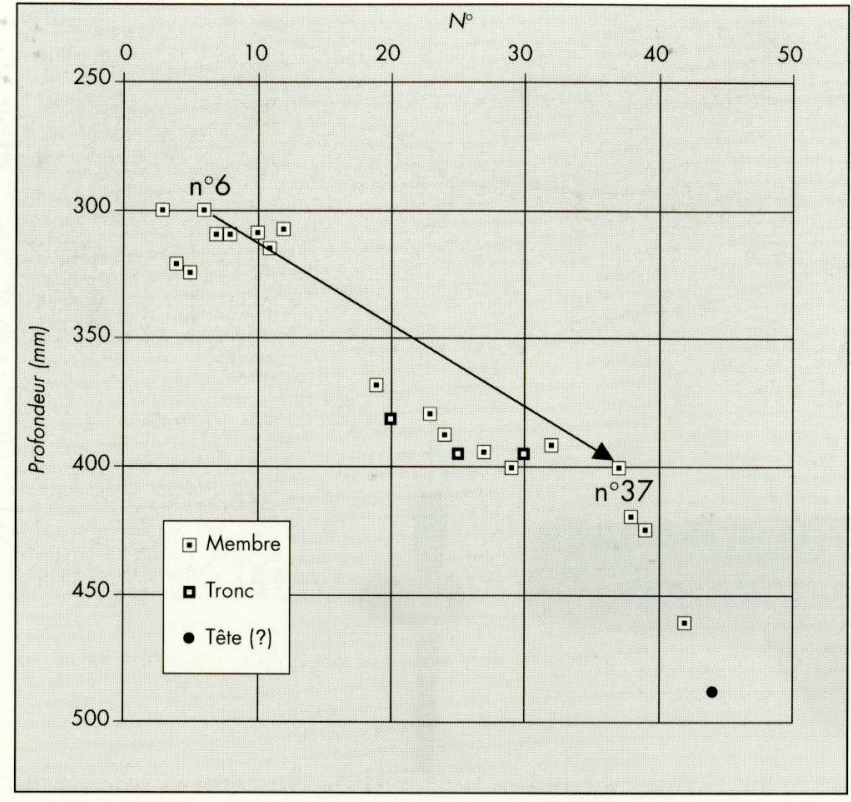

19 Distribution verticale des vestiges osseux humains (régions anatomiques et collage).

ra pas non plus un dépôt en deux temps car les vestiges sont trop peu nombreux, trop fragmentaires et surtout, la fouille n'a pas permis d'identifier deux couches distinctes. On remarquera enfin que les os rapportés au tronc et le possible fragment de crâne se situent dans la partie inférieure du loculus.

Tous les os, à l'exception d'un fragment de diaphyse $\left(n^{\circ} 5\right)$, présentent une couleur blanchâtre indiquant une température d'ustion supérieure à $650^{\circ} \mathrm{C}$. L'aspect de la fracturation renvoie à une crémation d'os frais.

Le dépôt d'os humains brûlés est très faible, et les comparaisons ne sont pas aisées car la datation de l'ensemble demeure sujette à révision. Cependant, on notera que des quantités tout aussi minimes et des indices pondéraux très proches ont été recensés dans des sépultures protohistoriques en Languedoc occidental, du Bronze final IIIB au $\mathrm{III}^{\mathrm{e}} \mathrm{s}$. av. n. è.

\section{Etude archéozoologique}

Le lot faunique étudié forme un assemblage majoritairement constitué de micro-fragments osseux (NR total $=113$ ), dont 70 sont carbonisés (avec un fragment dentaire), 2 sont brûlés à blanc et 41 ne sont pas brûlés. Les identifications permettent de restituer un ensemble faunique composé de :

- un humérus d'ovin-caprin non brûlé (détermination sous réserve);

- un corps de côte appartenant à un animal de petite taille (ovin, caprin ou chien) dont les fragments sont parfois brûlés; - un corps de côte (portion sous-articulaire) appartenant à un animal de taille moyenne (cervidé ou bovidé de petite taille)

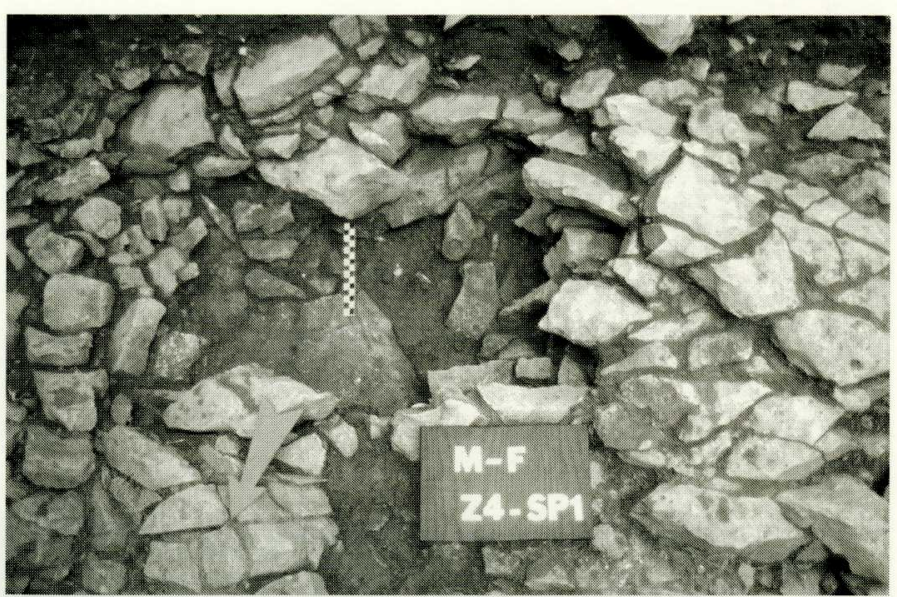

110 Extrémité distale de fémur de cerf rognée.

en partie carbonisé et dont un fragment porte une entaille de découpe au couteau sur la face médullaire de l'os compact au niveau de la diaphyse (découpe transverse de la côte); - une extrémité distale de phalange intermédiaire (phalange II) d'ovin-caprin non brûlée mais altérée et fissurée, sans doute sous l'action de la chaleur (feu indirect);

- une première molaire supérieure gauche de chien adulte (taille moyenne) brûlée (action du feu);

- une extrémité distale de fémur de cerf rognée (fig. 10).

Il s'agit d'une extrémité distale de fémur droit de cerf. L'état de synostose de l'épiphyse indique qu'il s'agit d'un individu mature.

Le fragment osseux se limite en fait au condyle latéral et à la lèvre latérale de la trochlée. En effet, l'os a été rogné par un animal dont les "coups de dents" ont laissé des traces très nettes sur le condyle et la trochlée; on remarque que la spongiosa de l'épiphyse a été creusée, ce qui constitue également une marque typique de l'intervention d'un animal (probablement un chien). Parallèlement, on ne relève aucune trace d'intervention anthropique.

Ce type de dépôt, en contexte funéraire, appartient à la catégorie des dépôts isolés de faune par opposition à l'offrande alimentaire type, constituée d'une portion de viande dont on retrouve généralement les ossements en connexion. Néanmoins, ce dépôt s'individualise au sein des dépôts isolés par le fait qu'il a été rogné; il ne s'agit pas d'un os entier comme c'est souvent le cas, traité alors comme un objet plus que comme une réelle offrande alimentaire. Sa signification, à ce stade, nous échappe, d'autant plus qu'ici, l'os a été préalablement jeté ou livré à l'animal puis récupéré et déposé dans la tombe.

Ce lot d'ossements et de dents est le résultat de la fragmentation et de la carbonisation de plusieurs éléments dont certains ont dû être consommés (côtes) et d'autres simplement déposés (fémur de cerf, dent/crâne de chien) mais qui tous, de près ou de loin, sont en relation avec la pratique funéraire qui consiste à consommer, à offrir, puis à déposer avec 


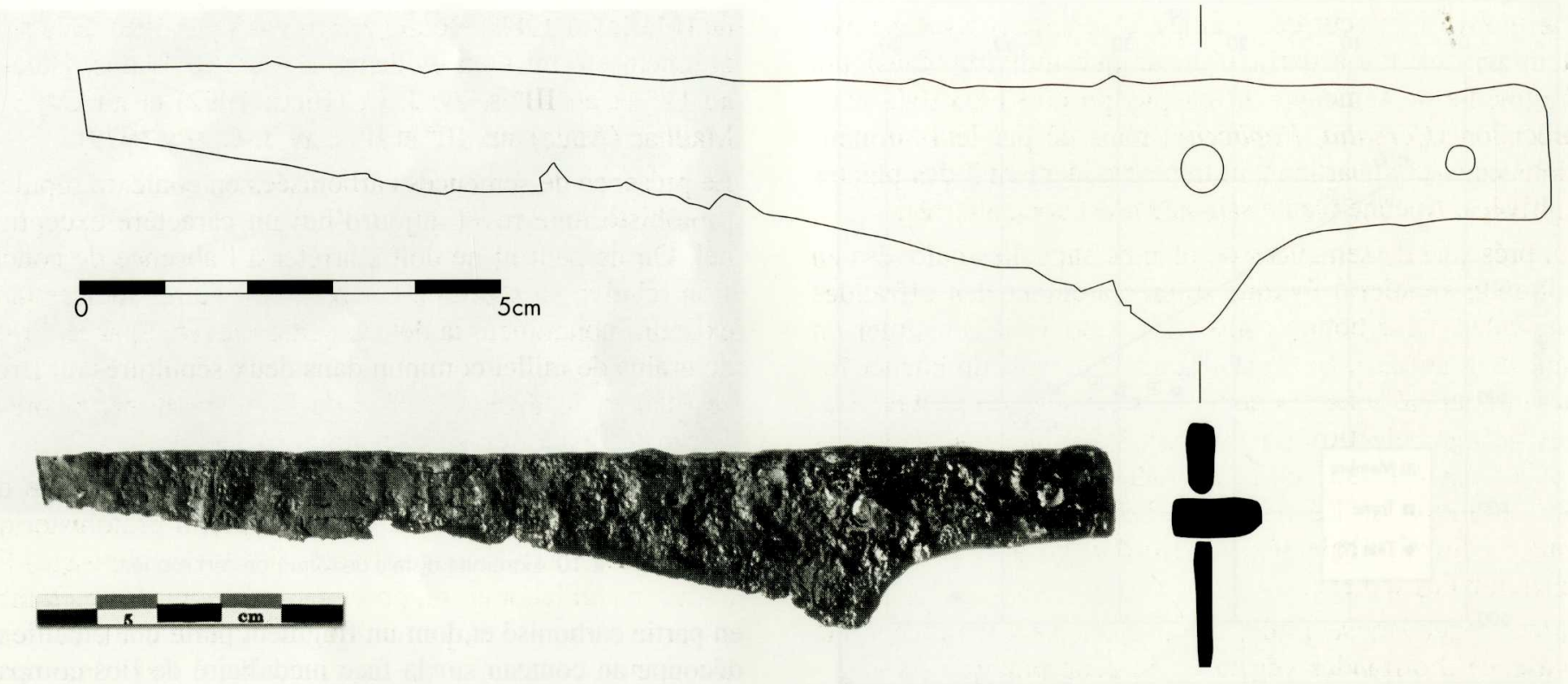

11 Dessin du couteau en fer et couteau restauré (cliché Loïc Damelet).

les restes du défunt des éléments liés au repas, aux sacrifices et aux offrandes.

\section{La céramique}

- Un fragment de panse d'amphore massaliète (environ 14 $\times 10 \mathrm{~cm}$ ).

- Quelques fragments informes de céramique non tournée qui ont subi l'action du feu.

\section{Le mobilier métallique}

- Un couteau en fer (fig. 11) ${ }^{4}$.

Couteau en fer complet, longueur conservée $17,3 \mathrm{~cm}$, largeur maximum de la lame $2,3 \mathrm{~cm}$, épaisseur maximum de la lame $0,3 \mathrm{~cm}$. Pointe légèrement cassée, dos droit, tranchant convergent au dos comportant des traces évidentes d'usure probablement liées à la durée d'utilisation de l'objet. Dans le prolongement de la lame, sans la moindre trace d'épaulement, une soie courte $(4,1 \mathrm{~cm})$ de section quadrangulaire aplatie à deux rivets espacés de $2,4 \mathrm{~cm}$. Sur cette soie nettement démarquée de la lame par un talon accentué, venait se fixer le manche en matière organique (bois, os ou corne). Etant donnée la position du rivet le plus proche de la lame, le manche devait recouvrir la totalité de la lame à sa largeur maximale.

\begin{tabular}{|c|c|c|c|}
\hline Nom latin & Nom commun & Type de reste & Nombre de restes \\
\hline \multirow{2}{*}{ Hordeum hexastichum } & Orge polystique vêtue & sem. ent. & 2 \\
\cline { 3 - 4 } & \multirow{2}{*}{ Blé nu } & fgt. & 3 \\
\hline Triticum aestivum/durum & Céréale & sem. ent. & 4 \\
\hline Cerealia & fgt. & 3 \\
\hline Lathyrus cicera/sativus & Gesse chiche/jarosse & sem. ent. & 7 \\
\hline Fabaceae & Légumineuse & fgt. & 1 \\
\hline
\end{tabular}

\section{Etude des carporestes}

Le tamisage à l'eau de l'intégralité du remplissage de la fosse sépulcrale a révélé à l'équipe archéologique la présence de restes carpologiques carbonisés. Ces éléments nous ont été transmis ainsi que les refus de tamis qui ont de ce fait pu être triés dans les conditions requises pour l'analyse carpologique, sous loupe binoculaire à grossissements variables selon la maille du tamis.

Le crible le plus fin utilisé lors du tamisage ne possède pas une ouverture inférieure à $1 \mathrm{~mm}$. Malgré sa finesse, ce mode d'échantillonnage ne garantit pas la récupération de tous les carporestes potentiellement présents. Il n'est pas par exemple exclu que certaines adventices ou des grains de millets aient échappé au tamisage.

La conservation des semences carbonisées est bonne, l'érosion des surfaces étant en général peu marquée. La fragmentation est relativement importante mais les cassures montrent qu'une part de celle-ci s'est produite sous l'action de l'eau au cours du tamisage.

Malgré leur nombre restreint les carporestes collectés permettent l'attestation de trois plantes domestiques (fig. 12). Il s'agit de deux céréales, le blé nu (Triticum aestivum/ durum) et l'orge polystique vêtue (Hordeum hexastichum), et d'une légumineuse, la gesse chiche/jarosse (Lathyrus cicera/sativus). Pour les semences archéologiques, le seul critère permettant la distinction de ces deux espèces de gesses cultivées est la Inventaire des carporestes. 
mesure de la largeur des graines. Une telle analyse est évidemment exclue à partir d'un unique individu. Plusieurs fragments de semences n'ont pas pu être identifiés avec précision (Cerealia, Fabaceae) mais de par leurs dimensions ceux-ci appartiennent très certainement à des plantes cultivées. Aucune plante sauvage n'est donc attestée.

La présence de semences de plantes annuelles cultivées en contexte sépulcral évoque immédiatement des offrandes végétales. Leur bonne conservation pourrait constituer un indice en ce sens. Si seuls certains éléments du bûcher funéraire ont été collectés pour être ensevelis, on peut envisager qu'une sélection ait favorisé les semences les mieux conservées à l'issue de la crémation. Malgré la faible représentativité de l'échantillon, l'absence de plantes sauvages pourrait également s'accorder à l'hypothèse d'une sélection des semences.

Il faut cependant se garder de conclure précipitamment à la présence d'offrandes végétales. Si cette pratique est largement attestée dans la Gaule romaine par la fréquence et l'abondance des restes végétaux recueillis dans les tombes (voir notamment Marinval 1993), elle demeure mal établie pour les époques antérieures du Bronze final et de l'âge du Fer (Marinval 1986; Janin et al. 1997). Il semble que, à ce jour, la seule description précise de semences carbonisées trouvées en abondance en contexte sépulcral protohistorique concerne les grains de millet commun étudiés par J. Erroux sur le site de Dampierre-sur-le-Doubs (Doubs). A l'intérieur d'une incinération primaire en fosse datée du Bronze final IIB, plusieurs litres de grains de millet ont été retrouvés sur le foyer, en association avec une meule fragmentée (Pétrequin et al. 1969; Pétrequin et al. 1985). Compte tenu du manque actuel de données dans ce domaine et du caractère limité de l'ensemble carpologique, on ne peut pas conclure à l'existence d'offrandes alimentaires à l'âge du Fer à Mourrel-Ferrat. Les semences carbonisées ont pu être introduites dans la fosse avec le sédiment de comblement ou s'infiltrer postérieurement au dépôt, d'autant que la sépulture se trouve en contexte d'habitat ou abondent souvent les déchets produits par les activités culinaires ou de traitement des récoltes.

D'un point de vue alimentaire et agricole, les trois plantes domestiques identifiées s'intègrent bien à nos connaissances sur l'économie languedocienne à l'âge du Fer. Le blé nu et l'orge polystique vêtue sont parmi les céréales les plus fréquentes à cette époque dans le Midi de la France. La prépondérance de l'orge vêtue est même un aspect essentiel de la céréaliculture tout au long de l'âge du Fer et ceci au moins depuis le Bronze final. La gesse chiche/jarosse est également une des légumineuses cultivées les mieux représentées sur les sites de la pério- de (Marinval 1988; Bouby, Marinval à paraître). Elle á notamment été mise en évidence à Lattara (Lattes, Hérault) au IV et au III ${ }^{\mathrm{e}}$ s. av. J.-C. (Buxo 1992) et au Cayla de Mailhac (Aude) aux III et II ${ }^{\mathrm{e}}$ s. av. J.-C. (Py 1979).

La présence de semences carbonisées en contexte sépulcral protohistorique revêt aujourd'hui un caractère exceptionnel. On ne peut ni ne doit s'arrêter à l'absence de conclusion relative au statut de ces restes. D'autres indices ténus existent, notamment la détermination récente par B. Pradat de grains de millet commun dans deux sépultures du Bronze final et du début de l'âge du Fer dans la nécropole du Camp d'Alba à Réalville (Janin et al. 1997).

Ces premiers éléments doivent inciter à poursuivre des études carpologiques en contexte sépulcral protohistorique à partir d'échantillonnages fiables. Seuls des tamisages fins et des tris en laboratoire, portant sur de nombreuses tombes et sur plusieurs sites, permettront de conclure à la présence d'offrandes végétales ou, au contraire, à l'extrême rareté des restes carpologiques et donc, éventuellement, au caractère accidentel de leur présence. Il est illusoire de penser que des témoins aussi discrets que des grains de millet ou de rares grains de céréales à paille pourraient être aisément repérés en faisant l'économie d'une recherche spécifique.

\section{Analyses et conclusions}

\subsection{ARCHITECTURE ET SIGNALISATION}

Pour la sépulture 1, aucun système de couverture n'a été enregistré. Toutefois, l'analyse de la coupe (qui correspond en fait à la limite du sondage) (fig. 13) laisse envisager une probable couverture de pierres sur un monticule de terre.

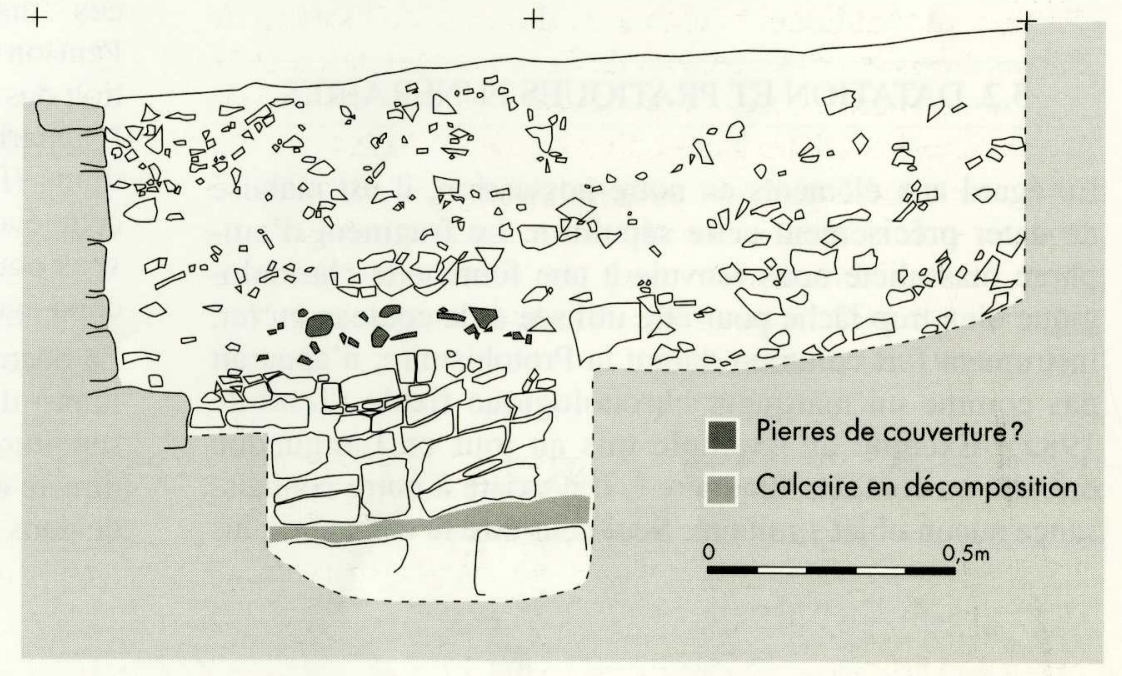

113 Probable couverture de pierres. 


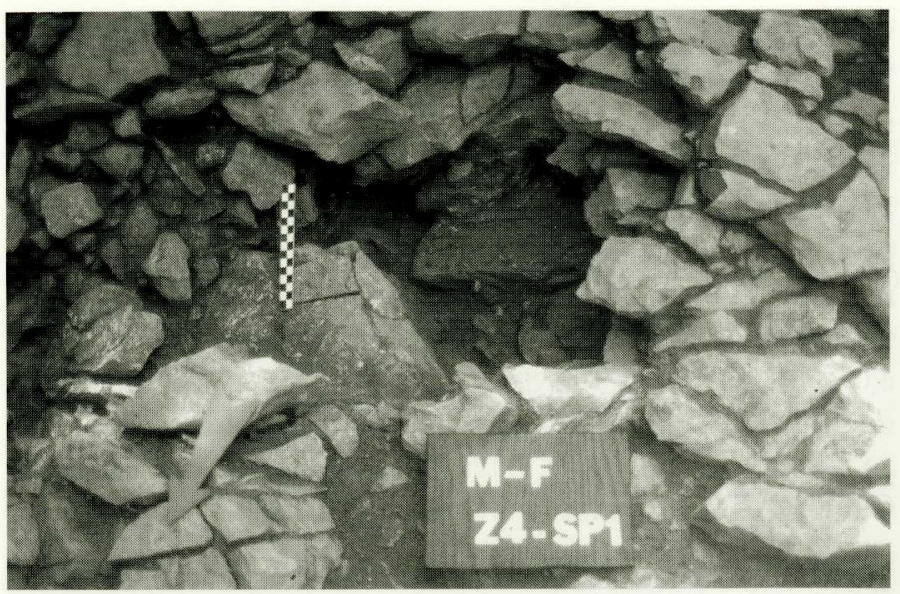

114 Loculus de la sépulture entièrement fouillé

Cette hypothèse, certes très fragile, pourra être vérifiée si l'ensemble 2 s'avère être effectivement une tombe. En effet, au milieu des blocs du substrat en place, des petites pierres dont une dalle forment un amas paracirculaire. Cette anomalie en contexte sépulcral nous fait penser à une possible signalisation tumulaire qu'il conviendra de discerner afin de repérer les sépultures.

En ce qui concerne l'architecture, la nature même du substrat génère des aménagements particuliers. En effet, l'enlèvement des blocs lors du creusement du loculus a entrâné la formation de "banquettes" ou d'étagères (fig. 14) dont la fonctionnalité n'a pu être réellement mise en évidence. S'il est difficile de parler de véritable organisation, la superposition du couteau sur le fragment d'amphore, luimême au-dessus de l'os de cerf, implique un aménagement interne du loculus et son remplissage. On peut imaginer que lors de la déposition des vestiges on a réalisé un colmatage progressif de la fosse avec le matériel décaissé, ou que des aménagements en matériaux périssables ont disparu et rendent difficile la lecture des données recueillies.

\subsection{DATATION ET PRATIQUES FUNÉRAIRES}

Eu égard aux éléments en notre possession, il est malaisé de dater précisément cette sépulture. Le fragment d'amphore massaliète nous renvoie à une fourchette chronologique bien trop lâche pour être utilisée et le couteau en fer, instrument fort commun durant la Protohistoire, n'apparaît pas comme un marqueur chronologique fiable (Tendille 1982). Excepté un exemple mis au jour en Languedoc oriental en contexte funéraire ${ }^{5}$, il n'existe à notre connaissance aucun objet similaire. Seul peut-être le caractère plus anguleux et saillant du talon qui isole la soie $\mathrm{du}_{\mathrm{i}}$ tranchant de la lame, rencontré sur certains couteaux à dos arqué du faciès Grand Bassin II, laisse entrevoir l'apparition de ce nouveau type de lame. Ce trait distinctif se retrouve également sur deux couteaux provenant d'une tombe à incinération du Mas Sainte-Puelles ${ }^{6}$ et sur quelques exemplaires découverts dans les tombes de la nécropole de Las Peyros à Couffoulens (Solier et al. 1976; Passelac et al. 1981). Si ces quelques éléments ne fournissent qu'une datation assez large, il est à noter que tous appartiennent au même horizon chronologique de la fin du $\mathrm{VI}^{\mathrm{e}} \mathrm{s}$. et de la première moitié du $\mathrm{V}^{\mathrm{e}}$ s. av. n. è. ${ }^{7}$

En confrontant ces résultats avec les données acquises sur les pratiques funéraires en Languedoc occidental (Rancoule 1984, 347-354; Rancoule 1989, 43-45), les caractéristiques de la sépulture 1 de Mourrel-Ferrat - absence d'ossuaire, forte fragmentation du mobilier d'accompagnement et architecture générale de la tombe - nous renvoient aux critères discriminants retenus pour cette période. Néanmoins, la présence de l'os de faune et le ramassage sélectif sur le bûcher funéraire sont autant de faits qui la distinguent.

En l'absence de critères chronologiques fiables, nous proposons de replacer la sépulture 1 de Mourrel-Ferrat dans le $\mathrm{V}^{\mathrm{e}} \mathrm{s}$. av. n. è. en privilégiant le caractère de la pratique funéraire même si elle reste encore mal connue pour cette période.

\section{Conclusion}

$\mathrm{L}$ a situation de cette tombe, datée probablement de la transition Fer I-Fer II, lui confère un caractère particulier. En effet, si SP1 peut évoquer la présence d'une nécropole sur l'oppidum de Mourrel-Ferrat, nous serions alors dans un cas comparable à celui d'Ensérune. La rareté de ces "installations" funéraires méritait d'être soulignée. Peut-on y voir un changement dans les choix d'implantation des sépultures traditionnellement localisées en plaine? L'apparition des tombes "de chef" situées au sein d'oppidums (Pech Maho, Mailhac) n'est-elle pas un reflet de cette évolution? Cela tendrait à expliquer les carences relevées pour les nécropoles de transition du premier et du second âges du Fer ainsi que pour les $\mathrm{IV}^{\mathrm{e}}$ et $\mathrm{III}^{\mathrm{e}} \mathrm{s}$. av. n. è.

La découverte récente (mars 1999, Janin et al. dans ce volume) d'un complexe funéraire - bûcher de crémation et sépultures - du dernier quart du $\mathrm{IV}^{\mathrm{e}} \mathrm{s}$. av. n. è. à proximité directe de l'enceinte de l'oppidum de Mourrel-Ferrat abonde dans ce sens et ouvre de séduisantes perspectives. 


\section{Notes de commentaire}

* UMR 154, Lattes-Montpellier.

** Centre d'Anthropologie, Toulouse.

1 Les principaux articles concernant l'oppidum de Mourrel-Ferrat (Olonzac, Hérault) sont donnés en bibliographie.

2 Nous tenons à remercier les membres du CRDM (Centre de Recherche et de Documentation du Minervois) ainsi que la municipalité d'Olonzac pour leur aide et leurs encouragements. Je n'oublie pas Annie Montécinos et Jean-Vincent Garribal, étudiants bénévoles, qui ont donné de leur temps et de leur patience pour la fouille de cette sépulture.

3 De nombreux conseils m'ont été prodigués par Martine Schwaller (SRA, Languedoc-Roussillon) et Thierry Janin (UMR 154, Lattes-Montpellier) lors de leur passage sur le terrain pendant la fouille. Que ces quelques lignes leur témoignent ma reconnaissance.

4 La restauration du couteau en fer a été confiée au Laboratoire de Restauration dirigé par Monique Drieux à Toulouse.

5 Un couteau (en fer, pointe manquante, soie d'emmanchement courte à 2 rivets, longueur conservée $164 \mathrm{~mm}$, largeur conservée $24 \mathrm{~mm}$ ) très proche du couteau de la sépulture 1 de Mourrel-Ferrat a été mis au jour dans la tombe 1 de la nécropole de la Bergerie Hermet (Calvisson, Gard) (Dedet, Py 1973, 40-42, fig. $4 \mathrm{n}^{\circ} 4$ ). Le mobilier d'accompagnement, coupe attique de type $C$ et un vase en céramique grise monochrome, permet une datation autour de 500 av. n. è. Cet exemplaire a été repris dans Tendille 1982,46 et 48 , fig. 8 $\mathrm{n}^{\circ} 48$.

6 Soutou, Vézian 1964, 164-172 et fig. $1 \mathrm{n}^{\circ} 3$ et $4:$ couteau en fer à soie plate munie de deux rivets de même métal, dont l'un est conservé; la lame présente un dos légèrement arqué et une pointe effilée. Longueur totale $230 \mathrm{~mm}$, largeur à la base de la lame $36 \mathrm{~mm}$. Couteau en fer de même forme que le précédent. Longueur conservée $162 \mathrm{~mm}$, largeur maximum de la lame $22 \mathrm{~mm}$.

$7 \mathrm{La}$ découverte récente d'un couteau lors de la fouille en laboratoire de l'ossuaire de la sépulture 422 de la nécropole du Moulin à Mailhac (Aude, fouille Th. Janin, UMR 154, Lattes-Montpellier) élargit encore la fourchette chronologique. En effet, l'exemplaire récemment étudié présente des caractéristiques similaires dans la morphologie et dans les dimensions à celui de la tombe 1 de Mourrel-Ferrat. Il provient d'une sépulture de faciès Grand-Bassin I (VII ${ }^{e}$ s. av. n. è.) (renseignements fournis par Th. Janin et N. Chardenon).

\section{Références bibliographiques}

Bouby, Marinval à paraître : BOUBY (L.), MARINVAL (P.) - Ressources végétales à Marseille et dans les sociétés indigènes au Bronze final et au premier Age du Fer : Premiers éléments de comparaison. In : Actes du colloque "Mailhac et le premier Age du Fer en Europe occidentale".

Buxo 1992 : BUXO I CAPDEVILA (R.) - Cueillette et agriculture à Lattes : les ressources végétales d'après les semences et les fruits. In : PY (M.) dir. - Recherches sur l'économie vivrière des Lattarenses. Lattes, ARALO, 1992, pp. 45-90 (Lattara, 5).

CRDM 1980 : Centre de Recherche et de Documentation du Minervois - Dégagement d'une poterne sur l'oppidum du Mourrel-Ferrat, Olonzac (Hérault). BSocAude, LXXX, 1980, pp. 15-20.

CRDM 1987 : Centre de Recherche et de Documentation du Minervois - Sondage sur l'oppidum du Mourrel-Ferrat à Olonzac (Hérault). La cabane P.G.1. ALanguedoc, 1987 (1.2.3), pp. 31-38.

Dedet, Py 1973 : DEDET (B.), PY (M.) - Les tombes protohistoriques de la Bergerie Hermet à Calvisson (Gard). Gallia, 31, 1973, pp. 37-59.

Duday 1989 : DUDAY (H.) - La nécropole du Peyrou à Agde (Hérault). Etude anthropologique. I $n$ : NICKELS (A.), MARCHAND (G.), SCHWALLER (M.) - Agde, la nécropole du Premier Age du fer. Paris, CNRS, 1989, 498 p. (suppl. à la RANarb, 19).

Janin et al. 1997 : JANIN (T.), BURENS (A.), CARROZA (L.) dir. - La nécropole protohistorique du Camp d'Alba à Réalville (Tarn-et-Garonne), ARALO-Archives d'Ecologie Préhistorique, Lattes-Toulouse, 1997. $174 \mathrm{p}$.

Jannoray 1955 : JANNORAY (J.) - Ensérune, contribution à l'étude des civilisations préromaines de la Gaule méridionale. Paris, de Boccard, 1955, $490 \mathrm{p}$. et $71 \mathrm{pl}$. (BEFAR, 181).

Marinval 1986 : MARINVAL (P.) - Présence de macro-restes végétaux (semences et fruits) en contexte funéraire protohistorique français. Anthropologie physique et Archéologie. Paris, CNRS, 1986, pp. 333-338.

Marinval 1988 : MARINVAL (P.) - Cueillette, agriculture et alimentation végétale de l'Epipaléolithique jusqu'au second Age du Fer en Grance méridionale : apports paléoethnographiques de la carpologie. Mémoire de thèse, EHESS, Paris, 1988.

Marinval 1993 : MARINVAL (P.) - Etude carpologique d'offrandes alimentaires végétales dans les sépultures gallo-romaines : réflexions préliminaires. In : FERDIÈRE (A.) dir. - Monde des morts, monde des vivants en Gaule rurale (I ${ }^{e r}$ s. av. J.-C. - Ve s. ap. J.-C.). Tours, 1993, pp. 45-65 (suppl. à la $R A C F r, 6$ ).
Mathieu 1931 : MATHIEU (L.) - Petite étude sur les vestiges de l'époque gallo-romaine dans la partie orientale du Minervois, BSocAude, XXXV, 1931, p. 265.

Passelac et al. 1981 : PASSELAC (M.), RANCOULE (G.), SOLIER (Y.) - La nécropole de Las Peyros à Couffoulens (Aude) (découverte d'un second groupe de tombes), RANarb, XIV, 1981, pp. 1-70.

Pétrequin et al. 1969 : PÉTREQUIN (P.), URLACHER (J.-P.), VUAILLAT (D.) - Habitat et sépultures de l'Age du bronze final à Dampierre-sur-leDoubs (Doubs), GalliaPrHist, XII, 1, 1969, pp. 1-35.

Pétrequin et al. 1985 : PÉTREQUIN (P.), CHAIX (L.), PETREQUIN (A.-M.), PININGRE (J.-F.) - La grotte des Planches-Près-Arbois (Jura). ProtoCortaillod et Age du Bronze Final. Paris, MSH, 1985.

Py 1979 : PY (M.) - Agriculture et artisanat. L'essor économique du $\mathrm{III}^{\mathrm{e}}$ au $\mathrm{I}^{\mathrm{er}}$ s. Dossiers de l'Archéologie, 35, 1979, pp. 86-98.

Rancoule 1984 : RANCOULE (G.) - Le bassin moyen de L'Aude à l'Age du Fer. Etat de la recherche, acquis et problèmes. Thèse de doctorat $\mathrm{III}^{e}$ cycle, Toulouse, 1984, 489 p. (dactyl.).

Rancoule 1989 : RANCOULE (G.) - Usages funéraires dans l'Aude au premier Age du fer et au début du second. DocAMérid, 12, 1989, pp. 41-49.

Schwaller et al. 1995 : SCHWALLER (M.), DUDAY (H.), JANIN (Th.), MARCHAND (G.), GARDEISEN (A.) - Cinq tombes du deuxième Age du fer à Ensérune (Nissan-lez-Ensérune, Hérault). In : Sur les pas des Grecs en Occident. Hommages à A. Nickels. Lattes/Paris, ADAM/Errance, 1995, pp. 205-230 (Et. Massa., 4)

Solier 1968 : SOLIER (Y.) - Une tombe de chef à l'oppidum de Pech Maho (Sigean, Aude). RANarb, 1, 1968, pp. 7-37.

Solier et al. 1976 : SOLIER (Y.), RANCOULE (G.), PASSELAC (M.) - La nécropole de Las Peyros, VI ${ }^{\mathrm{e}} \mathrm{s}$. av. J.-C. à Couffoulens (Aude). Paris, CNRS, 1976, 123 p. (suppl. à la RANarb, 6).

Soutou, Vézian 1964 : SOUTOU (A.), VEZIAN (J.) - Mobilier d'une tombe à incinération de Mas-Saintes-Puelles (Aude), CahLig, XIII, 1964, pp. 164-172.

Taffanel 1960 : TAFFANEL (J. et O.) - Deux tombes de chefs à Mailhac (Aude). Gallia, XVIII, 1960, pp. 1-37.

Tendille 1982 : TENDILLE (C.) - Mobiliers métalliques protohistoriques de la région nîmoise : instruments et outils divers. DocAMérid, 5, 1982, pp. 33-52. 